\title{
Passive Synthetic Aperture Radar Imaging of Ground Moving Targets
}

\author{
Steven Wacks and Birsen Yazıcı \\ Department of Electrical, Computer and Systems Engineering, Rensselaer Polytechnic \\ Institute, Troy, NY 12180 USA
}

\begin{abstract}
In this paper we present a method for imaging ground moving targets using passive synthetic aperture radar. A passive radar imaging system uses small, mobile receivers that do not radiate any energy. For these reasons, passive imaging systems result in significant cost, manufacturing, and stealth advantages. The received signals are obtained by multiple airborne receivers collecting scattered waves due to illuminating sources of opportunity such as commercial television, radio, and cell phone towers. We describe a novel forward model and a corresponding filtered-backprojection type image reconstruction method combined with entropy optimization. Our method determines the location and velocity of multiple targets moving at different velocities. Furthermore, it can accommodate arbitrary imaging geometries. we present numerical simulations to verify the imaging method.
\end{abstract}

\section{INTRODUCTION}

Since synthetic aperture radar (SAR) methods are typically designed for imaging stationary scenes, reconstructing scenes with moving targets is a challenging task. These moving targets appear smeared and unfocused in the reconstructed image. Many methods have been presented in the literature to address the problem of SAR imaging of ground moving targets (SAR/GMTI). ${ }^{1-10}$ The advantages of SAR and GMTI radar complement each other well: the image formation process can be used to help detect moving targets, and the target detection process can be used to help focus the image.

In this paper, we consider multiple passive airborne receivers to image both the stationary scene and moving targets. Figure 1 illustrates the imaging geometry considered. Passive radar systems provide many important advantages over active monostatic or bistatic imaging modalities. ${ }^{11-16}$ A passive radar system does not employ a dedicated transmitter by relying on illuminators of opportunity such as radio, cell phone, and television transmission towers. The absence of active signal propagation from the system provides key advantages such as cost, simplicity of implementation, and stealth.

We present a novel forward model and passive image formation method to reconstruct both the scene reflectivity and the two-dimensional velocity of multiple moving targets. A fully passive ground moving target SAR imaging method is attractive because it has the advantages of both categories of radar described above. Image formation, wide field of view, and high revisit rates benefit moving target detection, accurate motion parameter estimation can help better focus the image of scene reflectivity, and at the same time, system complexity is reduced with the elimination of transmitters and longevity is potentially increased with a more covert system.

Literature on passive SAR imaging can be found in. ${ }^{15,16} \mathrm{In},{ }^{15}$ an image reconstruction method is developed using filtered backprojection (FBP) on data correlated between the receivers to image a stationary scene. Similarly, ${ }^{16}$ also presents a method for passive SAR imaging of a stationary scene, but uses ultra-narrowband waveforms of opportunity.

In this paper, we extended the passive SAR imaging method introduced in $^{15}$ to imaging ground moving targets. We present an FBP method combined with an entropy optimization to reconstruct focused images of the ground and to determine the velocity of moving targets. Similar to the approach in, ${ }^{17,18}$ we reconstruct

Further author information: (Send correspondence to B.Y.)

B.Y.: E-mail: yazici@ecse.rpi.edu, Telephone: +1(518)276 2905, Fax: +1(518)276 6261

S.W.:E-mail: wackss@rpi.edu

Algorithms for Synthetic Aperture Radar Imagery XIX, edited by Edmund G. Zelnio, Frederick D. Garber, Proc. of SPIE Vol. 8394, 83940E · C 2012 SPIE · CCC code: 0277-786X/12/\$18 - doi: 10.1117/12.918610 


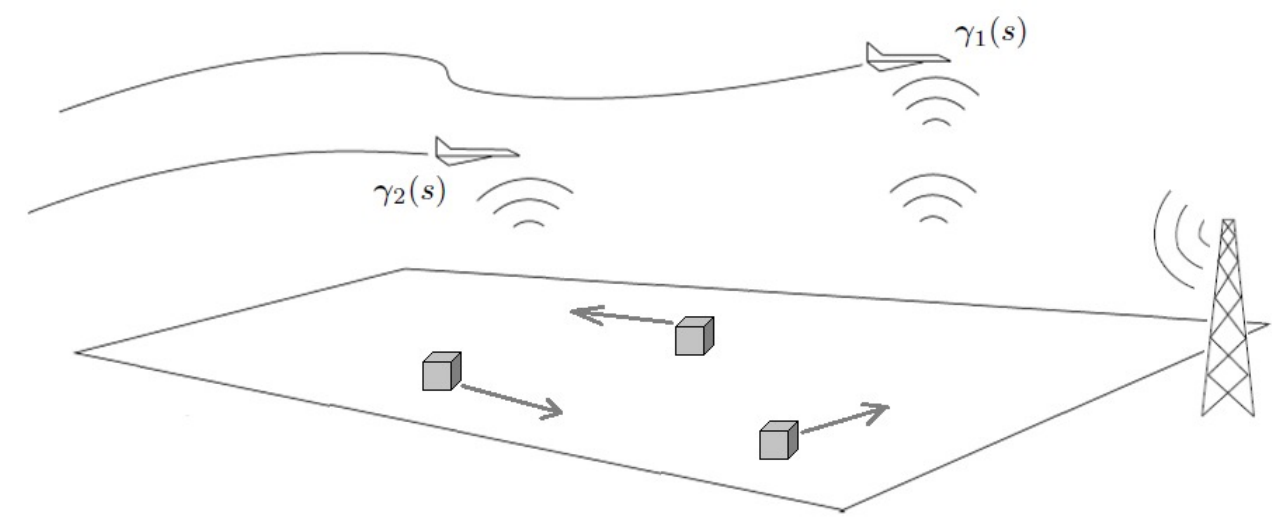

Figure 1: The imaging geometry considered in this paper. Multiple moving targets reflect the transmitted signal from an unknown, stationary transmitter to multiple passive receivers.

reflectivity images for a range of hypothesized velocities. We use entropy to measure the degree to which each reconstructed reflectivity image is focused. We estimate the target velocities by optimizing the entropy measure. Our algorithm has the ability to reconstruct the two-dimensional velocity of multiple moving targets.Finally, our work does not require prior information about the moving targets. The image stack and focus measure together provide the detection and estimation capability of moving targets and their motion parameters.

The organization of the remainder of the paper is as follows: In Section 2, we develop the forward model for SAH ground moving target imaging. In Section 3, we present an FBP-type image formation method for reconstructing the reflectivity of the scene for a given velocity and a entropy minimization technique for velocity estimation of multiple moving targets. In Section 4, we present numerical simulations to illustrate performance of our algorithm. Section 5 concludes the paper.

\section{FORWARD MODEL}

In this section a forward model is derived for a scene with ground moving targets and passive antennas. First, a model for ground moving targets will be derived. The model for the received field at each antenna will then be presented. Finally, the received signals from each pair of receiving antennas are correlated at each data processing interval.

We let $\mathbf{x}$ be a location on the ground where $\mathbf{x}=(\boldsymbol{x}, \psi(\boldsymbol{x})) \in \mathbb{R}^{3}, \boldsymbol{x} \in \mathbb{R}^{2}$, and $\psi: \mathbb{R}^{2} \rightarrow \mathbb{R}$ is a known function for the ground topography. Since the scattering takes place in a thin region near the surface and does not penetrate deep into the ground, the reflectivity function $V(\mathbf{x})$ is of the form

$$
V(\mathbf{x})=\rho(\boldsymbol{x}) \delta\left(x_{3}-\psi(\boldsymbol{x})\right)
$$

with $\rho(\boldsymbol{x})$ representing the surface reflectivity of a $2 \mathrm{D}$ point on the ground.

We now model the point scatterers, $\mathbf{z}$, as moving targets as a function of time. Without loss of generality, we let $\mathbf{x}$ be the position of the targets at the beginning of time $(s=0)$. Using an assumption of constant velocity for each moving target, we represent a scatterer's trajectory, $\mathbf{z}(s): \mathbb{R} \rightarrow \mathbb{R}^{3}$, by

$$
\mathbf{z}(s)=\mathbf{x}+\mathbf{v}_{\mathbf{x}} s
$$

where $\mathbf{v}_{\mathbf{x}}$ is the velocity of a particular point scatterer located at point $\mathbf{x}$ when $t=0$. Since the targets are all located on the ground, and we have a known function of the ground topography, the velocity of the scatters $\mathbf{v}_{\mathbf{x}}$ has the form

$$
\mathbf{v}_{\mathbf{x}}=\left[\boldsymbol{v}_{\boldsymbol{x}}, \nabla_{\boldsymbol{x}} \psi(\boldsymbol{x}) \cdot \boldsymbol{v}_{\boldsymbol{x}}\right]
$$

where $\nabla_{\boldsymbol{x}} \psi(\boldsymbol{x})=\left[\partial_{x_{1}} \psi(\boldsymbol{x}) \partial_{x_{2}} \psi(\boldsymbol{x})\right]$ is the 2D gradient of the ground topography. 
We extend the monostatic model introduced in ${ }^{19}$ for a stationary scene to one with a bistatic receiver configuration and combine our model of ground moving targets. We obtain the following for the received field at a single receiver $\gamma_{i}$ originating from a single, stationary transmitter $\mathbf{y}$ :

$$
f_{i}(s, t)=\int e^{-i \omega\left[t-\left(\left|\mathbf{x}-\gamma_{i}(s)\right|+|\mathbf{x}-\mathbf{y}|\right) / c_{0}-\left(\left(\widehat{\mathbf{x}-\boldsymbol{\gamma}_{i}(s)}\right)+\widehat{(\mathbf{x - y})}\right) \cdot \mathbf{v}_{\mathbf{x}} s / c_{0}\right]} \rho(\boldsymbol{x}) A(\omega, \boldsymbol{x}, s) d \omega d \boldsymbol{x}
$$

where $A(\omega, \boldsymbol{x}, s)$ is

$$
A(\omega, \boldsymbol{x}, s)=\frac{J_{R}(\omega, \boldsymbol{x}, s) J_{T}(\omega, \boldsymbol{x})}{(4 \pi)^{2}\left|\mathbf{x}-\gamma_{i}(s)\right||\mathbf{x}-\mathbf{y}|}
$$

and includes the waveform in $J_{T}$, the beamshaping factors in $J_{T}$ and $J_{R}$, and geometric attenuation factors in the denominator.

Using (4) as our model for the signal received by each receiving antenna, the cross-correlation of each pair of signals for each data processing window can now be computed in the following way:

$$
f_{i j}(s, \tau)=\int f_{i}(s, t) f_{j}^{*}(s, t-\tau) d t
$$

where $t$ is the fast-time variable and $t \in[0, T]$ with $T$ representing the length of the data processing window.

Under a series of assumptions, the expected value of the correlated received signal can be modeled as

$$
\begin{aligned}
d_{i j}(s, \tau)=E\left[f_{i j}(s, \tau)\right]:= & \mathcal{F}\left[R_{\rho}\right](s, \tau) \\
=\int & e^{-i \omega\left[\tau-R_{i j}(\mathbf{x}, s) / c_{0}-B_{i j}\left(\mathbf{x}, \mathbf{v}_{\mathbf{x}}, s\right) / c_{0}\right]} \\
& \times A_{i j}(\omega, s, \boldsymbol{x}) R_{\rho}(\boldsymbol{x}) d \boldsymbol{x} d \omega
\end{aligned}
$$

where

$$
\begin{aligned}
R_{i j}(\mathbf{x}, s) & =\left|\mathbf{x}-\gamma_{i}(s)\right|-\left|\mathbf{x}-\gamma_{j}(s)\right| \\
B_{i j}\left(\mathbf{x}, \mathbf{v}_{\mathbf{x}}, s\right) & =\left[\left(\mathbf{x}-\boldsymbol{\gamma}_{i}(s)\right)-\left(\mathbf{x}-\boldsymbol{\gamma}_{j}(s)\right)\right] \cdot \mathbf{v}_{\mathbf{x}} s \\
A_{i j}(\omega, s, \boldsymbol{x}) & =\frac{J_{R}(\omega, s, \boldsymbol{x}) J_{R}^{*}\left(\omega, s, \boldsymbol{x}^{\prime}\right)}{\left(4 \pi^{2}\right)\left|\mathbf{x}-\boldsymbol{\gamma}_{i}(s)\right|\left|\mathbf{x}^{\prime}-\gamma_{j}(s)\right|} \frac{R_{T}(\omega, \boldsymbol{x})}{(4 \pi)^{2}|\mathbf{x}-\mathbf{y}|^{2}}
\end{aligned}
$$

and $R_{\rho}(\boldsymbol{x})$ and $R_{T}(\boldsymbol{x})$ are the scene radiance and transmitter irradiance, respectively. ${ }^{15}$ In this model $R_{i j}$ is the hitchhiker range introduced in, ${ }^{15}$ and $B_{i j}$ is the component of the target displacement due to motion onto the hitchhiker look direction.

We assume that there is some $m_{A}$ such that $A$ satisfies

$$
\sup _{(\omega, s, \boldsymbol{x}) \in \mathcal{U}}\left|\partial_{\omega}^{\alpha} \partial_{s}^{\beta} \partial_{x_{1}}^{\rho_{1}} \partial_{x_{2}}^{\rho_{2}} A(\omega, s, \boldsymbol{x})\right| \leq C_{A}\left(1+\omega^{2}\right)^{\left(m_{A}-|\alpha|\right) / 2}
$$

where $\mathcal{U}$ is any compact subset of $\mathbb{R} \times \mathbb{R} \times \mathbb{R}^{2}$ and $C_{A}$ depends on $\mathcal{U}, \alpha, \beta, \rho_{1}$, and $\rho_{2}$. This assumption is needed to make various stationary phase approximations, and in practice, is satisfied when the antennas are sufficiently far away from the illuminated scene.

We refer to $\mathcal{F}$ as the forward operator for the $(i, j)$ pair of receivers. 


\section{IMAGE FORMATION}

The goal for image formation is to reconstruct the scene so that our image is simultaneously focused in both position and velocity spaces. Since the data in hand is two-dimensional, it may not be possible to form a focused image by backprojecting to the four-dimensional position and velocity space. Therefore, we backproject the correlated data onto the two-dimensional position space curves defined by the set $\left\{\boldsymbol{x}: R_{i j}(\mathbf{x}, s)+B_{i j}\left(\mathbf{x}, \mathbf{v}_{h}, s\right)=\right.$ $\left.\tau c_{0}, A_{i j}(\omega, s, \boldsymbol{x}) \neq 0\right\}$ for a fixed, hypothesized velocity $\mathbf{v}_{h}$.

In order to reconstruct an image of the scene, we will first filter backproject each pair of correlated signals with a hypothesized velocity. The filter will be designed such that the image is optimally focused when the hypothesized velocity is equal to the true velocity. These reconstructed images will then be coherently summed over all receiver pairs to form a $2 \mathrm{D}$ image of the scene radiance.

\subsection{Filtered Backprojection Operator}

The backprojection operator for a given pair of receivers is

$$
\begin{aligned}
\mathcal{K}\left[d_{i j}(s, \tau)\right]\left(\boldsymbol{z}, \boldsymbol{v}_{h}\right)= & \hat{R}_{\rho, i j}^{\boldsymbol{v}_{h}}(\boldsymbol{z}) \\
=\int & \int e^{i \omega\left[\tau-R_{i j}(\mathbf{z}, s) / c_{0}-B_{i j}\left(\mathbf{z}, \mathbf{v}_{h}, s\right) / c_{0}\right]} \\
& \times d_{i j}(s, \tau) Q_{i j}\left(\omega, s, \boldsymbol{z}, \boldsymbol{v}_{h}\right) d \omega d s d \tau
\end{aligned}
$$

where $Q_{i j}\left(\omega, s, \boldsymbol{z}, \boldsymbol{v}_{h}\right)$ is the filter to be determined later. $\mathbf{v}_{h}$ is a hypothesized velocity for which the image will be reconstructed. This is a velocity field and can have different values at different points in the image, allowing multiple moving targets with different velocities to be reconstructed.

Our reconstructed image will be the sum of all pairs of correlated data

$$
\begin{aligned}
& \hat{R}_{\rho}^{\boldsymbol{v}_{h}}(\boldsymbol{z})= \sum_{i, j} \hat{R}_{\rho, i j}^{\boldsymbol{v}_{h}}(\boldsymbol{z}) \\
&=\sum_{i, j} \int e^{i \omega\left[\tau-R_{i j}(\mathbf{z}, s) / c_{0}-B_{i j}\left(\mathbf{z}, \mathbf{v}_{h}, s\right) / c_{0}\right]} \\
& \quad \quad \times d_{i j}(s, \tau) Q_{i j}\left(\omega, s, \boldsymbol{z}, \boldsymbol{v}_{h}\right) d \omega d s d \tau
\end{aligned}
$$

and we assume that the filter $Q_{i j}$ satisfies a similar assumption to (9).

We now choose the filter $Q_{i j}$ such that the point spread function of the imaging operator for each pair of receivers is approximately the Dirac delta function under the assumption that the hypothesized velocity $\mathbf{v}_{h}$ equals the true velocity:

$$
\begin{aligned}
L_{i j}\left(\boldsymbol{x}, \boldsymbol{z}, \boldsymbol{v}_{h}, \boldsymbol{v}_{h}\right) & \approx \delta(\boldsymbol{x}-\boldsymbol{z}) \\
& =\int e^{i(\boldsymbol{x}-\boldsymbol{z}) \cdot \boldsymbol{\xi}} d \boldsymbol{\xi} .
\end{aligned}
$$

To arrive at the form of the point spread function in equation (12) we make a Taylor series approximation in the phase about the point $\boldsymbol{x}=\boldsymbol{z}$, and we make the following change of variables:

$$
(\omega, s) \rightarrow \boldsymbol{\xi}_{i j}=\left.\frac{\omega}{c_{0}} \nabla_{\boldsymbol{x}}\left[-R_{i j}(\mathbf{x}, s)-B_{i j}(\mathbf{x}, \mathbf{v}, s)\right]\right|_{\boldsymbol{x}=\boldsymbol{z}}
$$

The point spread function becomes

$$
L_{i j}\left(\boldsymbol{x}, \boldsymbol{z}, \boldsymbol{v}, \boldsymbol{v}_{h}\right) \approx \int e^{i(\boldsymbol{x}-\boldsymbol{z}) \cdot \boldsymbol{\xi}_{i j}} A_{i j}\left(\boldsymbol{\xi}_{i j}, \boldsymbol{x}\right) Q_{i j}\left(\boldsymbol{\xi}_{i j}, \boldsymbol{z}, \boldsymbol{v}_{h}\right) \eta\left(\boldsymbol{\xi}_{i j}, \boldsymbol{x}, \boldsymbol{z}, \boldsymbol{v}, \boldsymbol{v}_{h}\right) d \boldsymbol{\xi}_{i j}
$$


where $\eta$ is the determinant of the Jacobian that comes from the change of variables (13):

$$
\eta\left(\boldsymbol{\xi}_{i j}, \boldsymbol{x}, \boldsymbol{z}, \boldsymbol{v}, \boldsymbol{v}_{h}\right)=\left|\frac{\partial(\omega, s)}{\partial \boldsymbol{\xi}_{i j}}\right|
$$

and

$$
\begin{aligned}
A_{i j}\left(\boldsymbol{\xi}_{i j}, \boldsymbol{x}\right) & =A_{i j}\left(\omega\left(\boldsymbol{\xi}_{i j}\right), s\left(\boldsymbol{\xi}_{i j}\right), \boldsymbol{x}\right) \\
Q_{i j}\left(\boldsymbol{\xi}_{i j}, \boldsymbol{z}, \boldsymbol{v}_{h}\right) & =Q_{i j}\left(\omega\left(\boldsymbol{\xi}_{i j}\right), s\left(\boldsymbol{\xi}_{i j}\right), \boldsymbol{z}, \boldsymbol{v}_{h}\right) .
\end{aligned}
$$

Therefore, since we desire our filter to cause the point spread function to be as close as possible to the Dirac delta function, the optimal filter will be

$$
Q_{i j}\left(\boldsymbol{\xi}_{i j}, \boldsymbol{z}, \boldsymbol{v}_{h}\right)=\frac{\chi_{\Omega} A_{i j}^{*}\left(\boldsymbol{\xi}_{i j}, \boldsymbol{z}\right)}{\eta\left(\boldsymbol{\xi}_{i j}, \boldsymbol{z}, \boldsymbol{z}, \boldsymbol{v}, \boldsymbol{v}_{h}\right)\left|A_{i j}\left(\boldsymbol{\xi}_{i j}, \boldsymbol{z}\right)\right|^{2}}
$$

where $\chi_{\Omega}$ is a smooth cut-off function to prevent division by zero, and $\Omega_{\boldsymbol{z}}$ is the data collection manifold at $\boldsymbol{z}$ defined as $\Omega_{\boldsymbol{z}}=\left\{\boldsymbol{\xi}_{i j}: A_{i j}\left(\boldsymbol{\xi}_{i j}, \boldsymbol{z}\right) \neq 0\right\}$.

\subsection{Velocity Estimation}

To estimate the correct hypothesized velocity to use for the filtered backprojection operation, we first backproject a series of images for a range of velocities. Images that are backprojected with an incorrect hypothesized velocity have smeared targets, while the image with the correct velocity will have a focused target. We measure the degree of focus of each image, and the images with the highest focus yield the correct velocity estimations.

The measure of focus we use in this method is minimum entropy, ${ }^{20,21}$ since the entropy increases as moving targets are smeared. Given an image reconstructed with hypothesized velocity $\boldsymbol{v}_{h}$ the entropy is calculated by

$$
E\left(\boldsymbol{v}_{h}\right)=-\sum_{\boldsymbol{z}} p\left(\hat{R}_{\rho, i j}^{\boldsymbol{v}_{h}}(\boldsymbol{z})\right) \log \left[p\left(\hat{R}_{\rho, i j}^{\boldsymbol{v}_{h}}(\boldsymbol{z})\right)\right]
$$

where $p$ is a normalized histogram for the image. Once the images with minimum entropy are determined, their corresponding velocities are used to reconstruct the final filtered backprojected images.

\subsection{Velocity Estimation and Image Reconstruction Algorithm}

The full algorithm for velocity estimation and image reconstruction is as follows:

1. Form the correlated data using equation (6) for each pair of receivers.

2. For a range of hypothesized velocities in both the row and column dimensions, form initial images by backprojecting and summing each pair of correlated data.

3. Divide the scene into $M \times M$ regions, and for each region in each image, compute the entropy.

4. For each region, assign to it the hypothesized velocity corresponding to the minimum entropy for that region.

5. Use the hypothesized velocity determined in the previous step for each region to produce the filtered backprojected image. 


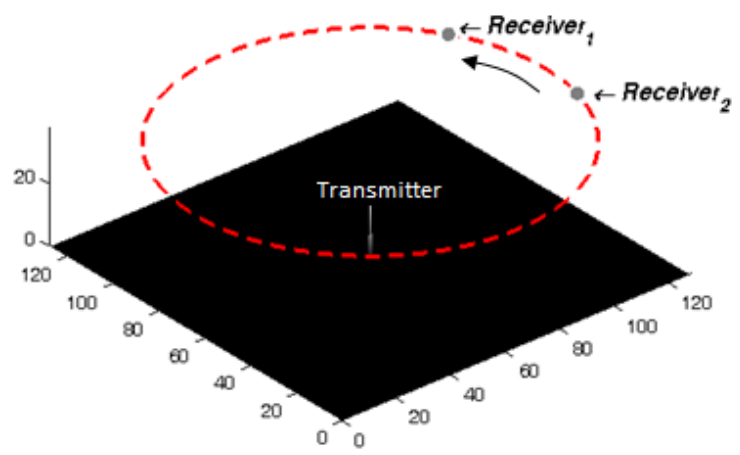

Figure 2: Setup for the simulations. The receivers move in a circular trajectory, while the transmitter is stationary in the center of the scene.

\section{NUMERICAL SIMULATIONS}

In this section we describe the numerical simulations that were performed in order to demonstrate the algorithm and its performance. Three simulations were created for different moving target scenarios. Scenes of size [22x22] $\mathrm{km}$ were constructed and discretized into [256x256] pixels. Three receiving antennas move in a circular aperture separated by a phase of $2 \pi / 3$ radians at a tangential velocity of about $270 \mathrm{~m} / \mathrm{s}$. The antennas each traverse a circular synthetic aperture discretized into 256 slow-time locations at an altitude of $5.5 \mathrm{~km}$. The transmitter is a stationary tower located in the center of the scene. A graphical illustration of this setup is shown in figure 2 .

The first simulation uses a single moving target that has a nonzero velocity in only one of its two components in order to test the most basic form of velocity estimation. The target is moving towards the right at a speed of $17 \mathrm{~m} / \mathrm{s}$. The scene at the initial time of $s=0$ is shown in figure 3 . Figure 4 shows the reconstructed image if a hypothesized velocity of zero is used. This incorrect velocity assumption produces a smeared target in the reconstructed image. The scene is then backprojected over the range of velocities from about [-43 -43] to [43 43] $\mathrm{m} / \mathrm{s}$ in increments of about $9 \mathrm{~m} / \mathrm{s}$. For each backprojected image, the entropy of the scene is calculated. Figure 5 shows a plot of the entropy for each hypothesized velocity. This method detects a clear minimum at the index 63 , which corresponds to the correct velocity of $17 \mathrm{~m} / \mathrm{s}$ towards the right. Finally, the reconstructed image for the scene is found by filtered backprojection with the $\left[\begin{array}{ll}17 & 0\end{array}\right] \mathrm{m} / \mathrm{s}$ as the hypothesized velocity and shown in figure 6 .

The second simulation uses a single moving target in the same starting location as the first simulation, but it has a nonzero velocity in both components. This target has a velocity of $[9-9] \mathrm{m} / \mathrm{s}$ which is in the direction of down and to the right. The original scene and reconstructed image for zero velocity assumption are shown in figures 7 and 8, respectively. The velocity estimation and image reconstruction was performed and the results are shown in figures 9 and 10. The entropy measure has a minimum at index 73 which corresponds to the correct velocity of $[9-9] \mathrm{m} / \mathrm{s}$.

The third simulation introduces a second moving target and shows how dividing the scene into regions allows the method to accurate estimate and reconstruct multiple moving targets. The original scene for this simulation is shown in figure 11. There are two moving targets, one in the top left moving to the right at a velocity of $9 \mathrm{~m} / \mathrm{s}$, and one in the bottom right moving at a velocity of $[-99] \mathrm{m} / \mathrm{s}$, which is towards the top left of the image. Figures 12 and 13 show the entropy functions for the two regions of the scene containing the moving targets. The first plot shows a minimum at index 62 , corresponding to a velocity $\left[\begin{array}{ll}9 & 0\end{array}\right] \mathrm{m} / \mathrm{s}$, and the second plot shows a minimum of 49 , corresponding to a velocity of [-9 9] m/s. Figure 14 shows the reconstructed image with the hypothesized velocity for each region of the image determined by the minimum entropy in the corresponding region. Some artifacts appear in this image as a result of incorrectly hypothesized velocities in regions with no target. This algorithm could be extended by preceding the processing with a target detection technique, which would help mitigate these artifacts. The reconstructed image does, however, clearly reconstruct both of the moving targets in their original locations at the beginning of the aperture. 


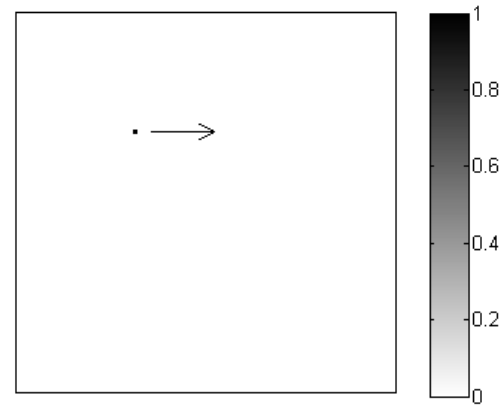

Figure 3: Original scene for simulation 1. There is one moving target located in the upper left quadrant of the scene with a velocity of $[170] \mathrm{m} / \mathrm{s}$.

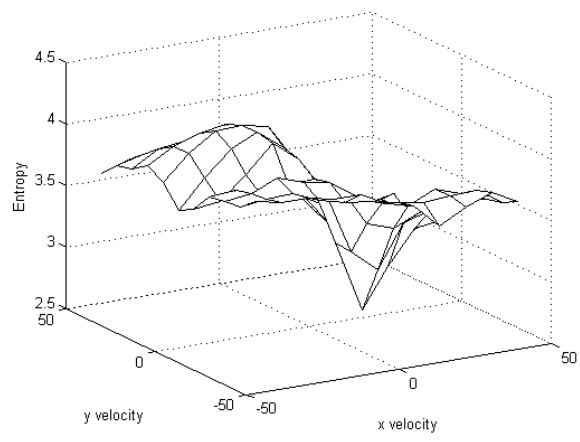

Figure 5: Entropy of the first scene plotted as a function of hypothesized velocity. The minimum point is at the $2 \mathrm{D}$ velocity $[170] \mathrm{m} / \mathrm{s}$.

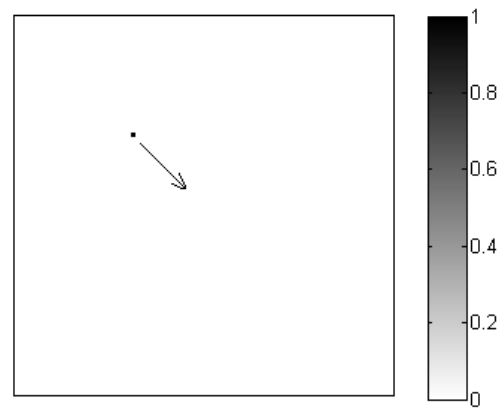

Figure 7: Original scene for simulation 2. There is one moving target located in the upper left quadrant of the scene with a velocity of $[9-9] \mathrm{m} / \mathrm{s}$.

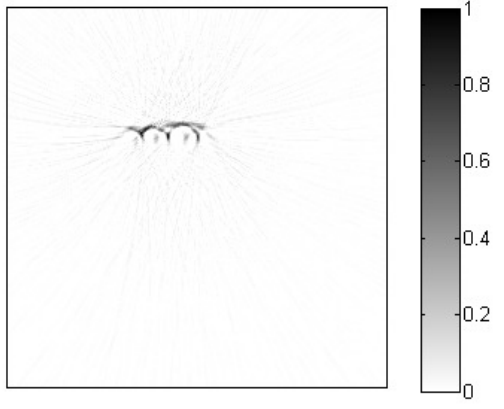

Figure 4: Scene 1 reconstructed with a hypothesized velocity of $0 \mathrm{~m} / \mathrm{s}$. Since the true velocity of the target is $17 \mathrm{~m} / \mathrm{s}$ to the right, the target appears smeared in the reconstructed image.

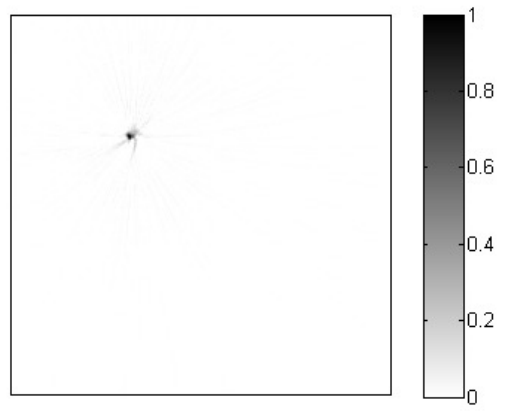

Figure 6: The reconstructed image for scene 1 with the hypothesized velocity for the image set to the true velocity vector estimated from minimum entropy.

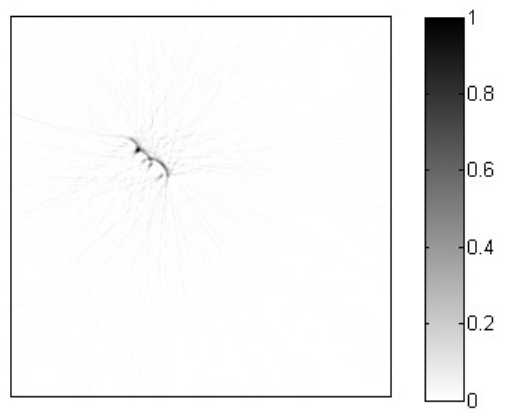

Figure 8: Scene 2 reconstructed with a hypothesized velocity of $0 \mathrm{~m} / \mathrm{s}$. Since the true velocity of the target is $[9-9] \mathrm{m} / \mathrm{s}$, the target appears smeared in the reconstructed image. 


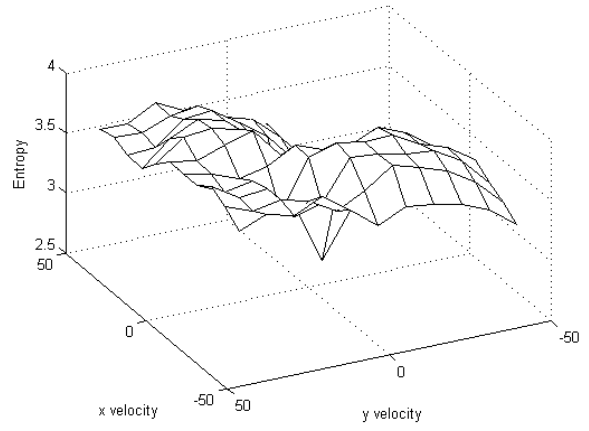

Figure 9: Entropy of the second scene plotted as a function of hypothesized velocity. The minimum point is at the $2 \mathrm{D}$ velocity $[9-9] \mathrm{m} / \mathrm{s}$.

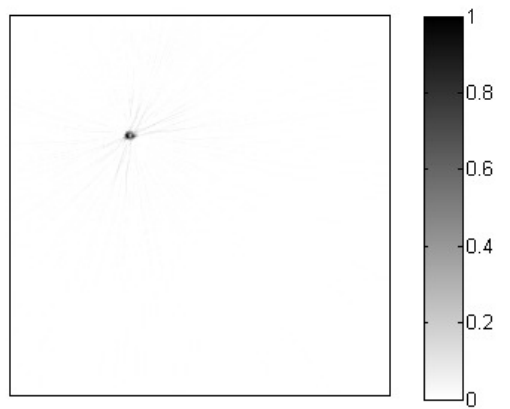

Figure 10: The reconstructed image for scene 2 with the hypothesized velocity for the image set to the true velocity vector estimated from minimum entropy.

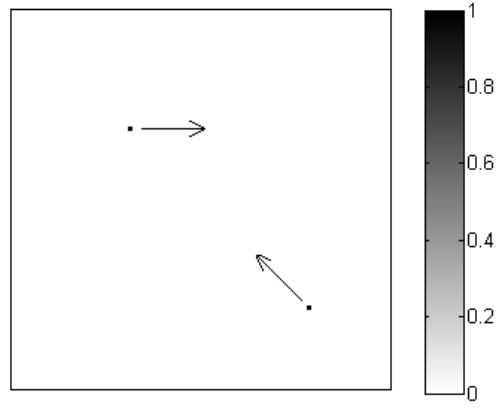

Figure 11: Original scene for simulation 3. There are two moving targets, one located in the upper left quadrant of the scene with velocity $\left[\begin{array}{ll}9 & 0\end{array}\right] \mathrm{m} / \mathrm{s}$ and one in the lower right quadrant moving at $\left[\begin{array}{ll}-9 & 9\end{array}\right] \mathrm{m} / \mathrm{s}$

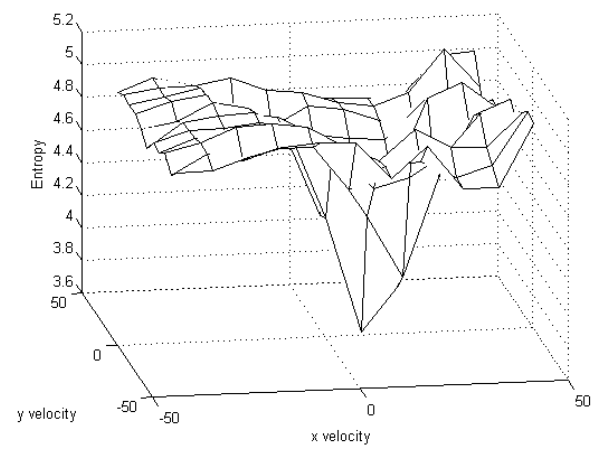

Figure 12: Entropy of the region of the third scene containing the first moving target. The minimum point is at the $2 \mathrm{D}$ velocity $[90] \mathrm{m} / \mathrm{s}$.

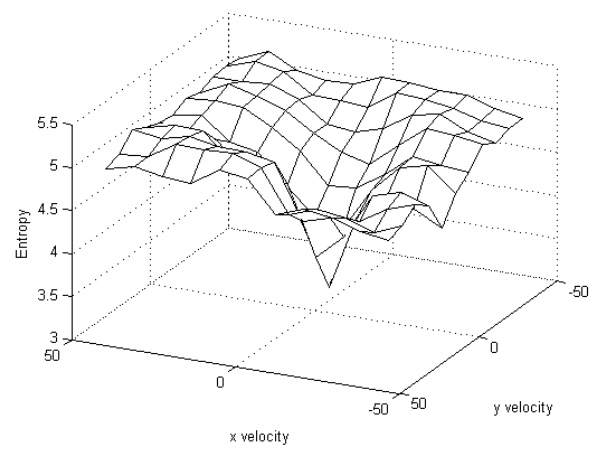

Figure 13: Entropy of the region of the third scene containing the second moving target. The minimum point is at the $2 \mathrm{D}$ velocity $[-99] \mathrm{m} / \mathrm{s}$. 


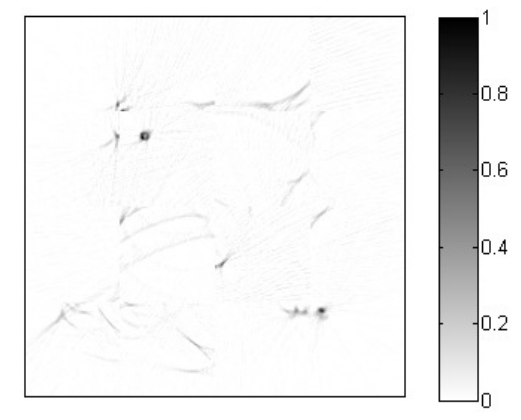

Figure 14: The reconstructed image for scene 3 with the hypothesized velocity for the image set to the true velocity vector estimated from minimum entropy.

\section{CONCLUSION}

In this paper we presented a method for image reconstruction and velocity estimation of a scene with moving targets using a network of passive receivers. Passive radar systems are an attractive solution because of their inherent cost, simplicity, and stealth advantages. Systems that are free of transmitters are cheaper to implement and harder to detect, which potentially increases the system's longevity. In addition, the problem of imaging moving targets is important to address, since targets of interest are often mobile, and without special processing they appear smeared and unfocused in the reconstructed image.

First, the paper introduces a novel forward model and inversion scheme for a moving scene. The forward model includes a modification of the phase for moving target displacement and then correlates pairs of received signals to remove transmitter information from the phase. The inversion scheme then reconstructs an image for a hypothesized velocity by using a filtered backprojection technique. Velocity estimation and image reconstruction are performed simultaneously by iteratively backprojecting images with different hypothesized velocities to determine the velocity corresponding to minimum entropy. Finally, numerical simulations were performed to verify the performance of the method.

In the future we plan on further investigating and enhancing the techniques introduced in this paper. A target detection step prior to velocity estimation may mitigate the artifacts when reconstructing images with multiple moving targets. In addition, resolution analysis for both position and velocity of targets would provide further insight into the accuracy and robustness of the algorithm presented here.

\section{Acknowledgement}

This work was supported by the Air Force Office of Scientific Research (AFOSR) under the agreements FA955007-1-0363 and FA9550-09-1-0013, and by the National Science Foundation (NSF) under Grant No. CCF08030672 .

\section{REFERENCES}

1. R. Deming, "Along-track interferometry for simultaneous sar and gmti: application to gotcha challenge data," in Proceedings of SPIE, 8051, p. 80510P, 2011.

2. V. Chen, "Time-frequency analysis of sar images with ground moving targets," in Proceedings of SPIE, 3391, p. 295, 1998.

3. M. Minardi, L. Gorham, and E. Zelnio, "Ground moving target detection and tracking based on generalized sar processing and change detection," in Proceedings of SPIE, 5808, p. 156, 2005.

4. D. Hack and M. Saville, "Analysis of sar moving grid processing for focusing and detection of ground moving targets," in Proceedings of SPIE, 8051, p. 80510S, 2011. 
5. S. Barbarossa, "Detection and imaging of moving objects with synthetic aperture radar. 1. optimal detection and parameter estimation theory," in Radar and Signal Processing, IEE Proceedings F, 139, pp. 79-88, IET, 1992.

6. M. Kirscht, "Detection and imaging of arbitrarily moving targets with single-channel sar," in Radar, Sonar and Navigation, IEE Proceedings-, 150, pp. 7-11, IET, 2003.

7. M. Stuff, M. Biancalana, G. Arnold, and J. Garbarino, "Imaging moving objects in 3d from single aperture synthetic aperture radar," in Radar Conference, 2004. Proceedings of the IEEE, pp. 94-98, IEEE, 2004.

8. F. Zhou, R. Wu, M. Xing, and Z. Bao, "Approach for single channel sar ground moving target imaging and motion parameter estimation," Radar, Sonar \& Navigation, IET 1(1), pp. 59-66, 2007.

9. R. Perry, R. Dipietro, and R. Fante, "Sar imaging of moving targets," Aerospace and Electronic Systems,

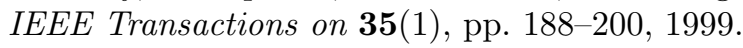

10. S. Zhu, G. Liao, Y. Qu, Z. Zhou, and X. Liu, "Ground moving targets imaging algorithm for synthetic aperture radar," Geoscience and Remote Sensing, IEEE Transactions on 49(1), pp. 462-477, 2011.

11. H. D. Griffiths and C. J. Baker, "Passive coherent location radar systems: part 1. performance prediction," IEE Proc., Radar Sonar Navig. 152, pp. 153-9, 2005.

12. A. D. Lanterman and J. D. C. Munson, "Deconvolution techniques for passive radar imaging," Algorithms for Synthetic Aperture Radar Imagery IX, Proc. SPIE 4727, pp. 166-177, 2002.

13. M. Çetin and A. Lanterman, "Region-enhanced imaging for sparse-aperture passive radar," Algorithms for Synthetic Aperture Radar Imagery XI, Proc. SPIE 5427, 2004.

14. Y. Wu and J. D. C. Munson, "Multistatic synthetic aperture imaging of aircraft using reflected television signals," Algorithms for Synthetic Aperture Radar Imagery VIII, Proc. SPIE 4382, 2001.

15. C. E. Yarman and B. Yazıcı, "Synthetic aperture hitchhiker imaging," IEEE Transactions on Imaging Processing 17(11), pp. 2156-2173, 2008.

16. C. E. Yarman, L. Wang, and B. Yazıcı, "Doppler synthetic aperture hitchhiker imaging," Inverse Problems 26 (065006), 2010.

17. M. Martorella, F. Berizzi, and B. Haywood, "Contrast maximisation based technique for 2-d isar autofocusing," in Radar, Sonar and Navigation, IEE Proceedings-, 152(4), pp. 253-262, IET, 2005.

18. C. Jakowatz Jr, D. Wahl, and P. Eichel, "Refocus of constant-velocity moving targets in synthetic aperture radar imagery," in Proceedings of SPIE, 3370, p. 85, 1998.

19. J. Jao, "Theory of synthetic aperture radar imaging of a moving target," Geoscience and Remote Sensing, IEEE Transactions on 39(9), pp. 1984-1992, 2001.

20. L. Xi, L. Guosui, and J. Ni, "Autofocusing of isar images based on entropy minimization," Aerospace and Electronic Systems, IEEE Transactions on 35(4), pp. 1240-1252, 1999.

21. D. Atkinson, D. Hill, P. Stoyle, P. Summers, and S. Keevil, "Automatic correction of motion artifacts in magnetic resonance images using an entropy focus criterion," Medical Imaging, IEEE Transactions on 16(6), pp. 903-910, 1997. 